\title{
Behavior of a Competitive System of Second-Order Difference Equations
}

\author{
Q. Din, ${ }^{1}$ T. F. Ibrahim, ${ }^{2,3}$ and K. A. Khan ${ }^{4}$ \\ ${ }^{1}$ Department of Mathematics, Faculty of Basic and Applied Sciences, University of Poonch Rawalakot, Rawalakot 12350, Pakistan \\ ${ }^{2}$ Department of Mathematics, Faculty of Sciences and Arts (S.A.), King Khalid University, Abha, Sarat Abida 61914, Saudi Arabia \\ ${ }^{3}$ Department of Mathematics, Faculty of Science, Mansoura University, Mansoura 35516, Egypt \\ ${ }^{4}$ Department of Mathematics, University of Sargodha, Sargodha 40100, Pakistan
}

Correspondence should be addressed to Q. Din; qamar.sms@gmail.com

Received 9 March 2014; Revised 5 April 2014; Accepted 26 April 2014; Published 15 May 2014

Academic Editor: Maoan Han

Copyright (C) 2014 Q. Din et al. This is an open access article distributed under the Creative Commons Attribution License, which permits unrestricted use, distribution, and reproduction in any medium, provided the original work is properly cited.

We study the boundedness and persistence, existence, and uniqueness of positive equilibrium, local and global behavior of positive equilibrium point, and rate of convergence of positive solutions of the following system of rational difference equations: $x_{n+1}=$ $\left(\alpha_{1}+\beta_{1} x_{n-1}\right) /\left(a_{1}+b_{1} y_{n}\right), y_{n+1}=\left(\alpha_{2}+\beta_{2} y_{n-1}\right) /\left(a_{2}+b_{2} x_{n}\right)$, where the parameters $\alpha_{i}, \beta_{i}, a_{i}$, and $b_{i}$ for $i \in\{1,2\}$ and initial conditions $x_{0}, x_{-1}, y_{0}$, and $y_{-1}$ are positive real numbers. Some numerical examples are given to verify our theoretical results.

\section{Introduction}

Systems of nonlinear difference equations of higher order are of paramount importance in applications. Such equations also appear naturally as discrete analogues and as numerical solutions of systems differential and delay differential equations which model diverse phenomena in biology, ecology, physiology, physics, engineering, and economics. For applications and basic theory of rational difference equations, we refer to [1-3]. In [4-10], applications of difference equations in mathematical biology are given. Nonlinear difference equations can be used in population models [11-17]. It is very interesting to investigate the behavior of solutions of a system of nonlinear difference equations and to discuss the local asymptotic stability of their equilibrium points.

Gibbons et al. [18] investigated the qualitative behavior of the following second-order rational difference equation:

$$
x_{n+1}=\frac{\alpha+\beta x_{n-1}}{\gamma+x_{n}} .
$$

Motivated by the above study, our aim in this paper is to investigate the qualitative behavior of positive solutions of the following second-order system of rational difference equations:

$$
x_{n+1}=\frac{\alpha_{1}+\beta_{1} x_{n-1}}{a_{1}+b_{1} y_{n}}, \quad y_{n+1}=\frac{\alpha_{2}+\beta_{2} y_{n-1}}{a_{2}+b_{2} x_{n}},
$$

where the parameters $\alpha_{i}, \beta_{i}, a_{i}$, and $b_{i}$ for $i \in\{1,2\}$ and initial conditions $x_{0}, x_{-1}, y_{0}$, and $y_{-1}$ are positive real numbers.

More precisely, we investigate the boundedness character, persistence, existence, and uniqueness of positive steady state, local asymptotic stability, and global behavior of unique positive equilibrium point and rate of convergence of positive solutions of system (2) which converge to its unique positive equilibrium point.

\section{Boundedness and Persistence}

The following theorem shows the boundedness and persistence of every positive solution of system (2).

Theorem 1. Assume that $\beta_{1}<a_{1}$ and $\beta_{2}<a_{2}$; then every positive solution $\left\{\left(x_{n}, y_{n}\right)\right\}$ of system (2) is bounded and persists. 
Proof. For any positive solution $\left\{\left(x_{n}, y_{n}\right)\right\}$ of system (2), one has

$$
\begin{array}{r}
x_{n+1} \leq A_{1}+B_{1} x_{n-1}, \quad y_{n+1} \leq A_{2}+B_{2} y_{n-1}, \\
n=0,1,2, \ldots,
\end{array}
$$

where $A_{i}=\alpha_{i} / a_{i}$ and $B_{i}=\beta_{i} / a_{i}$ for $i \in\{1,2\}$. Consider the following linear difference equations:

$$
\begin{aligned}
& u_{n+1}=A_{1}+B_{1} u_{n-1}, \quad n=0,1,2, \ldots, \\
& v_{n+1}=A_{2}+B_{2} v_{n-1}, \quad n=0,1,2, \ldots
\end{aligned}
$$

Obviously, solutions of these second-order nonhomogeneous difference equations are given by

$$
\begin{aligned}
& u_{n}=\frac{A_{1}}{1-B_{1}}+c_{1} B_{1}^{n / 2}+c_{2}\left(-\sqrt{B_{1}}\right)^{n}, \quad n=1,2, \ldots, \\
& v_{n}=\frac{A_{2}}{1-B_{2}}+c_{3} B_{2}^{n / 2}+c_{4}\left(-\sqrt{B_{2}}\right)^{n}, \quad n=1,2, \ldots,
\end{aligned}
$$

where $c_{i}$ for $i \in\{1,2,3,4\}$ depend upon initial conditions $u_{-1}$, $u_{0}, v_{-1}$, and $v_{0}$. Assume that $\beta_{1}<a_{1}$ and $\beta_{2}<a_{2}$; then the sequences $\left\{u_{n}\right\}$ and $\left\{v_{n}\right\}$ are bounded. Suppose that $u_{-1}=x_{-1}$, $u_{0}=x_{0}, v_{-1}=y_{-1}$, and $v_{0}=y_{0}$; then by comparison we have

$$
\begin{array}{r}
x_{n} \leq \frac{\alpha_{1}}{a_{1}-\beta_{1}}=U_{1}, \quad y_{n} \leq \frac{\alpha_{2}}{a_{2}-\beta_{2}}=U_{2}, \\
n=1,2, \ldots .
\end{array}
$$

Furthermore, from system (2) and (6) we obtain that

$$
\begin{aligned}
& x_{n+1} \geq \frac{\alpha_{1}}{a_{1}+b_{1} y_{n}} \geq \frac{\alpha_{1}\left(a_{2}-\beta_{2}\right)}{a_{1}\left(a_{2}-\beta_{2}\right)+b_{1} \alpha_{2}}=L_{1}, \\
& y_{n+1} \geq \frac{\alpha_{2}}{a_{2}+b_{2} x_{n}} \geq \frac{\alpha_{2}\left(a_{1}-\beta_{1}\right)}{a_{2}\left(a_{1}-\beta_{1}\right)+b_{2} \alpha_{1}}=L_{2} .
\end{aligned}
$$

From (6) and (7), it follows that

$$
L_{1} \leq x_{n} \leq U_{1}, \quad L_{2} \leq y_{n} \leq U_{2}, \quad n=1,2, \ldots
$$

Hence, theorem is proved.

Lemma 2. Let $\left\{\left(x_{n}, y_{n}\right)\right\}$ be a positive solution of system (2). Then, $\left[L_{1}, U_{1}\right] \times\left[L_{2}, U_{2}\right]$ is invariant set for system (2).

Proof. The proof follows by induction.

\section{Stability Analysis}

Let us consider fourth-dimensional discrete dynamical system of the following form:

$$
\begin{array}{r}
x_{n+1}=f\left(x_{n}, x_{n-1}, y_{n}, y_{n-1}\right), \\
y_{n+1}=g\left(x_{n}, x_{n-1}, y_{n}, y_{n-1}\right), \\
n=0,1, \ldots,
\end{array}
$$

where $f: I^{2} \times J^{2} \rightarrow I$ and $g: I^{2} \times J^{2} \rightarrow J$ are continuously differentiable functions and $I, J$ are some intervals of real numbers. Furthermore, a solution $\left\{\left(x_{n}, y_{n}\right)\right\}_{n=-1}^{\infty}$ of system (9) is uniquely determined by initial conditions $\left(x_{i}, y_{i}\right) \in I \times J$ for $i \in\{-1,0\}$. Along with system (9), we consider the corresponding vector map $F=\left(f, x_{n}, g, y_{n}\right)$. An equilibrium point of (9) is a point $(\bar{x}, \bar{y})$ that satisfies

$$
\begin{aligned}
& \bar{x}=f(\bar{x}, \bar{x}, \bar{y}, \bar{y}), \\
& \bar{y}=g(\bar{x}, \bar{x}, \bar{y}, \bar{y}) .
\end{aligned}
$$

The point $(\bar{x}, \bar{y})$ is also called a fixed point of the vector $\operatorname{map} F$.

Definition 3. Let $(\bar{x}, \bar{y})$ be an equilibrium point of the system (9).

(i) An equilibrium point $(\bar{x}, \bar{y})$ is said to be stable if for every $\varepsilon>0$ there exists $\delta>0$ such that, for every initial condition $\left(x_{i}, y_{i}\right), i \in\{-1,0\}$ if $\| \sum_{i=-1}^{0}\left(x_{i}, y_{i}\right)-$ $(\bar{x}, \bar{y}) \|<\delta$ implies that $\left\|\left(x_{n}, y_{n}\right)-(\bar{x}, \bar{y})\right\|<\varepsilon$ for all $n>0$, where $\|\cdot\|$ is usual Euclidian norm in $\mathbb{R}^{2}$.

(ii) An equilibrium point $(\bar{x}, \bar{y})$ is said to be unstable if it is not stable.

(iii) An equilibrium point $(\bar{x}, \bar{y})$ is said to be asymptotically stable if there exists $\eta>0$ such that

$$
\left\|\sum_{i=-1}^{0}\left(x_{i}, y_{i}\right)-(\bar{x}, \bar{y})\right\|<\eta,
$$

$$
\left(x_{n}, y_{n}\right) \longrightarrow(\bar{x}, \bar{y}) \quad \text { as } n \longrightarrow \infty \text {. }
$$

(iv) An equilibrium point $(\bar{x}, \bar{y})$ is called global attractor if $\left(x_{n}, y_{n}\right) \rightarrow(\bar{x}, \bar{y})$ as $n \rightarrow \infty$.

(v) An equilibrium point $(\bar{x}, \bar{y})$ is called asymptotic global attractor if it is a global attractor and stable.

Definition 4. Let $(\bar{x}, \bar{y})$ be an equilibrium point of a map $F=$ $\left(f, x_{n}, g, y_{n}\right)$, where $f$ and $g$ are continuously differentiable functions at $(\bar{x}, \bar{y})$. The linearized system of (9) about the equilibrium point $(\bar{x}, \bar{y})$ is

$$
X_{n+1}=F\left(X_{n}\right)=F_{J} X_{n},
$$

where $X_{n}=\left(\begin{array}{c}x_{n} \\ y_{n} \\ x_{n-1} \\ y_{n-1}\end{array}\right)$ and $F_{J}$ is Jacobian matrix of system (9) about the equilibrium point $(\bar{x}, \bar{y})$.

To construct the corresponding linearized form of system (2) we consider the following transformation:

$$
\left(x_{n}, y_{n}, x_{n-1}, y_{n-1}\right) \longmapsto\left(f, g, f_{1}, g_{1}\right) \text {, }
$$

where $f=x_{n+1}, g=y_{n+1}, f_{1}=x_{n}$, and $g_{1}=y_{n}$. The linearized system of (2) about $(\bar{x}, \bar{y})$ is given by

$$
Z_{n+1}=F_{J}(\bar{x}, \bar{y}) Z_{n}
$$


where $Z_{n}=\left(\begin{array}{c}x_{n} \\ y_{n} \\ x_{n-1} \\ y_{n-1}\end{array}\right)$ and the Jacobian matrix about the fixed point $(\bar{x}, \bar{y})$ under the transformation (13) is given by

$$
\begin{aligned}
& F_{J}(\bar{x}, \bar{y}) \\
& =\left(\begin{array}{cccc}
0 & -\frac{b_{1} \bar{x}}{a_{1}+b_{1} \bar{y}} & \frac{\beta_{1}}{a_{1}+b_{1} \bar{y}} & 0 \\
-\frac{b_{2} \bar{y}}{a_{2}+b_{2} \bar{x}} & 0 & 0 & \frac{\beta_{2}}{a_{2}+b_{2} \bar{x}} \\
1 & 0 & 0 & 0 \\
0 & 1 & 0 & 0
\end{array}\right) .
\end{aligned}
$$

Lemma 5. Assume that $X_{n+1}=F\left(X_{n}\right), n=0,1, \ldots$, is a system of difference equations such that $\bar{X}$ is a fixed point of $F$. If all eigenvalues of the Jacobian matrix $J_{F}$ about $\bar{X}$ lie inside the open unit disk $|\lambda|<1$, then $\bar{X}$ is locally asymptotically stable. If one of them has a modulus greater than one, then $\bar{X}$ is unstable.

The following theorem shows the existence and uniqueness of positive equilibrium point of system (2).

Theorem 6. Assume that $\beta_{1}<a_{1}$ and $\beta_{2}<a_{2}$; then there exists unique positive equilibrium point of system (2) in $\left[L_{1}, U_{1}\right] \times\left[L_{2}, U_{2}\right]$, if the following condition is satisfied:

$$
\begin{aligned}
& \alpha_{1} \alpha_{2} b_{1} b_{2} \\
& \quad<\left(a_{1} a_{2}+b_{2}\left(a_{1}-\beta_{1}\right) L_{1}-a_{1} \beta_{2}-a_{2} \beta_{1}+\alpha_{2} b_{1}+\beta_{1} \beta_{2}\right)^{2} .
\end{aligned}
$$

Proof. Consider the following system of equations:

$$
x=\frac{\alpha_{1}+\beta_{1} x}{a_{1}+b_{1} y}, \quad y=\frac{\alpha_{2}+\beta_{2} y}{a_{2}+b_{2} x} .
$$

Assume that $(x, y) \in\left[L_{1}, U_{1}\right] \times\left[L_{2}, U_{2}\right]$; then it follows from (17) that

$$
x=\frac{\alpha_{1}}{a_{1}-\beta_{1}+b_{1} y}, \quad y=\frac{\alpha_{2}}{a_{2}-\beta_{2}+b_{2} x} .
$$

Take

$$
F(x)=\frac{\alpha_{1}}{a_{1}-\beta_{1}+b_{1} f(x)}-x,
$$

where $f(x)=\alpha_{2} /\left(a_{2}-\beta_{2}+b_{2} x\right)$ and $x \in\left[L_{1}, U_{1}\right]$. Then, we obtain that

$$
f\left(L_{1}\right)=\frac{\alpha_{2}}{a_{2}-\beta_{2}}\left(\frac{a_{1}\left(a_{2}-\beta_{2}\right)+b_{1} \alpha_{2}}{a_{1}\left(a_{2}-\beta_{2}\right)+b_{1} \alpha_{2}+b_{2} \alpha_{1}}\right)<\frac{\alpha_{2}}{a_{2}-\beta_{2}} .
$$

Hence, it follows that

$$
\begin{aligned}
F\left(L_{1}\right)= & \frac{\alpha_{1}}{a_{1}-\beta_{1}+b_{1} f\left(L_{1}\right)}-L_{1} \\
> & \frac{\alpha_{1}\left(a_{2}-\beta_{2}\right)}{\left(a_{1}-\beta_{1}\right)\left(a_{2}-\beta_{2}\right)+b_{1} \alpha_{2}}-L_{1} \\
= & \frac{\alpha_{1}\left(a_{2}-\beta_{2}\right)}{\left(a_{1}-\beta_{1}\right)\left(a_{2}-\beta_{2}\right)+b_{1} \alpha_{2}} \\
& -\frac{\alpha_{1}\left(a_{2}-\beta_{2}\right)}{a_{1}\left(a_{2}-\beta_{2}\right)+b_{1} \alpha_{2}}>0 .
\end{aligned}
$$

Furthermore,

$$
\begin{aligned}
F\left(U_{1}\right) & =\frac{\alpha_{1}}{a_{1}-\beta_{1}+b_{1} f\left(U_{1}\right)}-U_{1} \\
& =\frac{\alpha_{1}}{a_{1}-\beta_{1}}\left(\frac{\left(a_{2}-\beta_{2}\right)\left(a_{1}-\beta_{1}\right)+\alpha_{1} b_{2}}{\left(a_{2}-\beta_{2}\right)\left(a_{1}-\beta_{1}\right)+\alpha_{1} b_{2}+b_{1} \alpha_{2}}-1\right) \\
& <0 .
\end{aligned}
$$

Hence, $F(x)=0$ has at least one positive solution in $\left[L_{1}, U_{1}\right]$.

Furthermore, assume that condition (16) is satisfied; then one has

$$
F^{\prime}(x)
$$

$$
\begin{gathered}
=\left(\alpha _ { 1 } \alpha _ { 2 } b _ { 1 } b _ { 2 } \left(\left(a_{1} a_{2}+a_{1} b_{2} x-a_{1} \beta_{2}-a_{2} \beta_{1}\right.\right.\right. \\
\left.\left.\left.+\alpha_{2} b_{1}+\beta_{1} \beta_{2}-\beta_{1} b_{2} x\right)^{2}\right)^{-1}\right)-1
\end{gathered}
$$$$
\leq\left(\alpha _ { 1 } \alpha _ { 2 } b _ { 1 } b _ { 2 } \left(\left(a_{1} a_{2}+b_{2}\left(a_{1}-\beta_{1}\right) L_{1}-a_{1} \beta_{2}\right.\right.\right.
$$

$$
\left.\left.\left.-a_{2} \beta_{1}+\alpha_{2} b_{1}+\beta_{1} \beta_{2}\right)^{2}\right)^{-1}\right)-1
$$

$<0$.

Hence, $F(x)=0$ has a unique positive solution in $\left[L_{1}, U_{1}\right]$. The proof is therefore completed.

Theorem 7. The unique positive equilibrium point $(\bar{x}, \bar{y})$ of system (2) is locally asymptotically stable if $b_{1} b_{2} U_{1} U_{2}+\beta_{1} \beta_{2}+$ $\beta_{1}\left(a_{2}+b_{2} L_{1}\right)+\beta_{2}\left(a_{1}+b_{1} L_{2}\right)<\left(a_{1}+b_{1} L_{2}\right)\left(a_{2}+b_{2} L_{1}\right)$.

Proof. The characteristic polynomial of Jacobian matrix $F_{J}(\bar{x}, \bar{y})$ about $(\bar{x}, \bar{y})$ is given by

$P(\lambda)$

$$
\begin{aligned}
= & \lambda^{4}-\left(\frac{b_{1} b_{2} \overline{x y}}{\left(a_{1}+b_{1} \bar{y}\right)\left(a_{2}+b_{2} \bar{x}\right)}+\frac{\beta_{1}}{a_{1}+b_{1} \bar{y}}+\frac{\beta_{2}}{a_{2}+b_{2} \bar{x}}\right) \lambda^{2} \\
& +\frac{\beta_{1} \beta_{2}}{\left(a_{1}+b_{1} \bar{y}\right)\left(a_{2}+b_{2} \bar{x}\right)} .
\end{aligned}
$$


Let $\Phi(\lambda)=\lambda^{4}$ and $\Psi(\lambda)=\left(\left(b_{1} b_{2} \overline{x y} /\left(a_{1}+b_{1} \bar{y}\right)\left(a_{2}+b_{2} \bar{x}\right)\right)+\right.$ $\left.\left(\beta_{1} /\left(a_{1}+b_{1} \bar{y}\right)\right)+\left(\beta_{2} /\left(a_{2}+b_{2} \bar{x}\right)\right)\right) \lambda^{2}-\left(\beta_{1} \beta_{2} /\left(a_{1}+b_{1} \bar{y}\right)\left(a_{2}+b_{2} \bar{x}\right)\right)$. Assume that $b_{1} b_{2} U_{1} U_{2}+\beta_{1} \beta_{2}+\beta_{1}\left(a_{2}+b_{2} L_{1}\right)+\beta_{2}\left(a_{1}+b_{1} L_{2}\right)<$ $\left(a_{1}+b_{1} L_{2}\right)\left(a_{2}+b_{2} L_{1}\right)$ and $|\lambda|=1$; then one has

$|\Psi(\lambda)|$

$$
\begin{aligned}
& <\left(\frac{b_{1} b_{2} \overline{x y}}{\left(a_{1}+b_{1} \bar{y}\right)\left(a_{2}+b_{2} \bar{x}\right)}+\frac{\beta_{1}}{a_{1}+b_{1} \bar{y}}+\frac{\beta_{2}}{a_{2}+b_{2} \bar{x}}\right) \\
& \quad+\frac{\beta_{1} \beta_{2}}{\left(a_{1}+b_{1} \bar{y}\right)\left(a_{2}+b_{2} \bar{x}\right)} \\
& <\frac{b_{1} b_{2} U_{1} U_{2}}{\left(a_{1}+b_{1} L_{2}\right)\left(a_{2}+b_{2} L_{1}\right)}+\frac{\beta_{1}}{a_{1}+b_{1} L_{2}} \\
& \quad+\frac{\beta_{2}}{a_{2}+b_{2} L_{1}}+\frac{\beta_{1} \beta_{2}}{\left(a_{1}+b_{1} L_{2}\right)\left(a_{2}+b_{2} L_{1}\right)} \\
& =\frac{b_{1} b_{2} U_{1} U_{2}+\beta_{1} \beta_{2}+\beta_{1}\left(a_{2}+b_{2} L_{1}\right)+\beta_{2}\left(a_{1}+b_{1} L_{2}\right)}{\left(a_{1}+b_{1} L_{2}\right)\left(a_{2}+b_{2} L_{1}\right)} \\
& <1 .
\end{aligned}
$$

Then, by Rouche's Theorem, $\Phi(\lambda)$ and $\Phi(\lambda)-\Psi(\lambda)$ have the same number of zeroes in an open unit disk $|\lambda|<1$. Hence, all the roots of (24) satisfy $|\lambda|<1$, and it follows from Lemma 5 that the unique positive equilibrium point $(\bar{x}, \bar{y})$ of the system (2) is locally asymptotically stable.

Arguing as in [2], we have following result for global behavior of (2).

Lemma 8. Assume that $f:(0, \infty) \times(0, \infty) \rightarrow(0, \infty)$ and $g$ : $(0, \infty) \times(0, \infty) \rightarrow(0, \infty)$ are continuous functions and $a, b$, $c$, and $d$ are positive real numbers with $a<b, c<d$. Moreover, suppose that $f:[a, b] \times[c, d] \rightarrow[a, b]$ and $g:[a, b] \times$ $[c, d] \rightarrow[c, d]$ such that following conditions are satisfied:

(i) $f(x, y)$ is increasing in $x$ and decreasing in $y$, and $g(x, y)$ is decreasing in $x$ and increasing in $y$;

(ii) let $m_{1}, M_{1}, m_{2}$, and $M_{2}$ be real numbers such that $m_{1}=f\left(m_{1}, M_{2}\right), M_{1}=f\left(M_{1}, m_{2}\right), m_{2}=g\left(M_{1}, m_{2}\right)$, and $M_{2}=g\left(m_{1}, M_{2}\right)$; then $m_{1}=M_{1}$ and $m_{2}=M_{2}$.

Then, the system of difference equations $x_{n+1}=$ $f\left(x_{n-1}, y_{n}\right), y_{n+1}=g\left(x_{n}, y_{n-1}\right)$ has a unique positive equilibrium point $(\bar{x}, \bar{y})$ such that $\lim _{n \rightarrow \infty}\left(x_{n}, y_{n}\right)=(\bar{x}, \bar{y})$.

Theorem 9. The unique positive equilibrium point of system (2) is global attractor if $\left(a_{1}-\beta_{1}+b_{1} L_{2}\right)^{2}\left(a_{2}-\beta_{2}+b_{1} L_{1}\right)^{2}>$ $\alpha_{1} \alpha_{2} b_{1} b_{2}$.

Proof. Let $f(x, y)=\left(\alpha_{1}+\beta_{1} x\right) /\left(a_{1}+b_{1} y\right)$ and $g(x, y)=\left(\alpha_{2}+\right.$ $\left.\beta_{2} y\right) /\left(a_{2}+b_{2} x\right)$. Then, it is easy to see that $f(x, y)$ is increasing in $x$ and decreasing in $y$. Moreover, $g(x, y)$ is decreasing in $x$ and increasing in $y$. Let $\left(m_{1}, M_{1}, m_{2}, M_{2}\right)$ be a solution of the system

$$
\begin{array}{ll}
m_{1}=f\left(m_{1}, M_{2}\right), & M_{1}=f\left(M_{1}, m_{2}\right), \\
m_{2}=g\left(M_{1}, m_{2}\right), & M_{2}=g\left(m_{1}, M_{2}\right) .
\end{array}
$$

Then, one has

$$
\begin{array}{ll}
m_{1}=\frac{\alpha_{1}+\beta_{1} m_{1}}{a_{1}+b_{1} M_{2}}, & M_{1}=\frac{\alpha_{1}+\beta_{1} M_{1}}{a_{1}+b_{1} m_{2}}, \\
m_{2}=\frac{\alpha_{2}+\beta_{2} m_{2}}{a_{2}+b_{2} M_{1}}, & M_{2}=\frac{\alpha_{2}+\beta_{2} M_{2}}{a_{2}+b_{2} m_{1}} .
\end{array}
$$

Furthermore, we have

$$
\begin{array}{ll}
L_{1} \leq m_{1}, & M_{1} \leq U_{1}, \\
L_{2} \leq m_{2}, & M_{2} \leq U_{2} .
\end{array}
$$

From (27), it follows that

$$
\begin{array}{ll}
m_{1}=\frac{\alpha_{1}}{a_{1}-\beta_{1}+b_{1} M_{2}}, & M_{1}=\frac{\alpha_{1}}{a_{1}-\beta_{1}+b_{1} m_{2}}, \\
m_{2}=\frac{\alpha_{2}}{a_{2}-\beta_{2}+b_{2} M_{1}}, & M_{2}=\frac{\alpha_{2}}{a_{2}-\beta_{2}+b_{2} m_{1}} .
\end{array}
$$

On subtracting (29), one has

$$
\begin{aligned}
M_{1} & -m_{1} \\
& =\alpha_{1}\left(\frac{1}{a_{1}-\beta_{1}+b_{1} m_{2}}-\frac{1}{a_{1}-\beta_{1}+b_{1} M_{2}}\right) \\
& =\frac{\alpha_{1} b_{1}\left(M_{2}-m_{2}\right)}{\left(a_{1}-\beta_{1}+b_{1} m_{2}\right)\left(a_{1}-\beta_{1}+b_{1} M_{2}\right)} \\
& \leq \frac{\alpha_{1} b_{1}\left(M_{2}-m_{2}\right)}{\left(a_{1}-\beta_{1}+b_{1} L_{2}\right)^{2}} .
\end{aligned}
$$

Similarly, from (30), we obtain

$$
M_{2}-m_{2} \leq \frac{\alpha_{2} b_{2}\left(M_{1}-m_{1}\right)}{\left(a_{2}-\beta_{2}+b_{1} L_{1}\right)^{2}} .
$$

Furthermore, from (31) and (32), we obtain

$$
\left(K-\alpha_{1} \alpha_{2} b_{1} b_{2}\right)\left(M_{1}-m_{1}\right) \leq 0,
$$

where $K=\left(a_{1}-\beta_{1}+b_{1} L_{2}\right)^{2}\left(a_{2}-\beta_{2}+b_{1} L_{1}\right)^{2}$. Finally, from (33), it follows that $m_{1}=M_{1}$. Similarly, it is easy to see that $m_{2}=M_{2}$.

Lemma 10. Under the conditions of Theorems 7 and 9 the unique positive equilibrium of (2) is globally asymptotically stable. 


\section{Rate of Convergence}

In this section, we will determine the rate of convergence of a solution that converges to the unique positive equilibrium point of the system (2).

The following result gives the rate of convergence of solutions of a system of difference equations:

$$
X_{n+1}=(A+B(n)) X_{n}
$$

where $X_{n}$ is an $m$-dimensional vector, $A \in C^{m \times m}$ is a constant matrix, and $B: \mathbb{Z}^{+} \rightarrow C^{m \times m}$ is a matrix function satisfying

$$
\|B(n)\| \longrightarrow 0
$$

as $n \rightarrow \infty$, where $\|\cdot\|$ denotes any matrix norm which is associated with the vector norm

$$
\|(x, y)\|=\sqrt{x^{2}+y^{2}} .
$$

Proposition 11 (Perron's Theorem, [19]). Suppose that condition (35) holds. If $X_{n}$ is a solution of (34), then either $X_{n}=0$ for all large $n$ or

$$
\rho=\lim _{n \rightarrow \infty}\left(\left\|X_{n}\right\|\right)^{1 / n}
$$

exists and is equal to the modulus of one of the eigenvalues of matrix $A$.

Proposition 12 (see [19]). Suppose that condition (35) holds. If $X_{n}$ is a solution of (34), then either $X_{n}=0$ for all large $n$ or

$$
\rho=\lim _{n \rightarrow \infty} \frac{\left\|X_{n+1}\right\|}{\left\|X_{n}\right\|}
$$

exists and is equal to the modulus of one of the eigenvalues of matrix $A$.

Let $\left\{\left(x_{n}, y_{n}\right)\right\}$ be an arbitrary solution of the system (2) such that $\lim _{n \rightarrow \infty} x_{n}=\bar{x}$ and $\lim _{n \rightarrow \infty} y_{n}=\bar{y}$, where $\bar{x} \epsilon$ $\left[L_{1}, U_{1}\right]$ and $\bar{y} \in\left[L_{2}, U_{2}\right]$. To find the error terms, one has from the system (2)

$$
\begin{aligned}
x_{n+1}-\bar{x} & =\frac{\alpha_{1}+\beta_{1} x_{n-1}}{a_{1}+b_{1} y_{n}}-\frac{\alpha_{1}+\beta_{1} \bar{x}}{a_{1}+b_{1} \bar{y}} \\
& =\frac{\beta_{1}\left(x_{n-1}-\bar{x}\right)}{a_{1}+b_{1} y_{n}}-\frac{b_{1} \bar{x}\left(y_{n}-\bar{y}\right)}{a_{1}+b_{1} y_{n}}, \\
y_{n+1}-\bar{y} & =\frac{\alpha_{2}+\beta_{2} y_{n-1}}{a_{2}+b_{2} x_{n}}-\frac{\alpha_{2}+\beta_{2} \bar{y}}{a_{2}+b_{2} \bar{x}} \\
& =-\frac{b_{2} \bar{y}\left(x_{n}-\bar{x}\right)}{a_{2}+b_{2} x_{n}}+\frac{\beta_{2}\left(y_{n-1}-\bar{y}\right)}{a_{2}+b_{2} x_{n}} .
\end{aligned}
$$

Let $e_{n}^{1}=x_{n}-\bar{x}$ and $e_{n}^{2}=y_{n}-\bar{y}$; then one has

$$
\begin{aligned}
& e_{n+1}^{1}=a_{n} e_{n-1}^{1}+b_{n} e_{n}^{2}, \\
& e_{n+1}^{2}=c_{n} e_{n}^{1}+d_{n} e_{n-1}^{2},
\end{aligned}
$$

where

$$
\begin{array}{ll}
a_{n}=\frac{\beta_{1}}{a_{1}+b_{1} y_{n}}, & b_{n}=-\frac{b_{1} \bar{x}}{a_{1}+b_{1} y_{n}}, \\
c_{n}=-\frac{b_{2} \bar{y}}{a_{2}+b_{2} x_{n}}, & d_{n}=\frac{\beta_{2}}{a_{2}+b_{2} x_{n}} .
\end{array}
$$

Moreover,

$$
\begin{array}{ll}
\lim _{n \rightarrow \infty} a_{n}=\frac{\beta_{1}}{a_{1}+b_{1} \bar{y}}, & \lim _{n \rightarrow \infty} b_{n}=-\frac{b_{1} \bar{x}}{a_{1}+b_{1} \bar{y}}, \\
\lim _{n \rightarrow \infty} c_{n}=-\frac{b_{2} \bar{y}}{a_{2}+b_{2} \bar{x}}, & \lim _{n \rightarrow \infty} d_{n}=\frac{\beta_{2}}{a_{2}+b_{2} \bar{x}} .
\end{array}
$$

Now, the limiting system of error terms can be written as

$$
\left[\begin{array}{c}
e_{n+1}^{1} \\
e_{n+1}^{2} \\
e_{n}^{1} \\
e_{n}^{2}
\end{array}\right]
$$$$
=\left(\begin{array}{cccc}
0 & -\frac{b_{1} \bar{x}}{a_{1}+b_{1} \bar{y}} & \frac{\beta_{1}}{a_{1}+b_{1} \bar{y}} & 0 \\
-\frac{b_{2} \bar{y}}{a_{2}+b_{2} \bar{x}} & 0 & 0 & \frac{\beta_{2}}{a_{2}+b_{2} \bar{x}} \\
1 & 0 & 0 & 0 \\
0 & 1 & 0 & 0
\end{array}\right)
$$$$
\times\left[\begin{array}{c}
e_{n}^{1} \\
e_{n}^{2} \\
e_{n-1}^{1} \\
e_{n-1}^{2}
\end{array}\right],
$$

which is similar to linearized system of (2) about the equilibrium point $(\bar{x}, \bar{y})$. Using Proposition 11, one has following result.

Theorem 13. Assume that $\left\{\left(x_{n}, y_{n}\right)\right\}$ is a positive solution of the system (2) such that $\lim _{n \rightarrow \infty} x_{n}=\bar{x}$ and $\lim _{n \rightarrow \infty} y_{n}=\bar{y}$, where $\bar{x} \in\left[L_{1}, U_{1}\right]$ and $\bar{y} \in\left[L_{2}, U_{2}\right]$. Then, the error vector $e_{n}=\left(\begin{array}{c}e_{n}^{1} \\ e_{n}^{2} \\ e_{n-1}^{1} \\ e_{n-1}^{2}\end{array}\right)$ of every solution of (2) satisfies both of the following asymptotic relations:

$$
\begin{gathered}
\lim _{n \rightarrow \infty}\left(\left\|e_{n}\right\|\right)^{1 / n}=\left|\lambda_{1,2,3,4} F_{J}(\bar{x}, \bar{y})\right|, \\
\lim _{n \rightarrow \infty} \frac{\left\|e_{n+1}\right\|}{\left\|e_{n}\right\|}=\left|\lambda_{1,2,3,4} F_{J}(\bar{x}, \bar{y})\right|,
\end{gathered}
$$

where $\lambda_{1,2,3,4} F_{J}(\bar{x}, \bar{y})$ are the characteristic roots of Jacobian matrix $F_{J}(\bar{x}, \bar{y})$. 


\section{Existence of Unbounded Solutions of (2)}

In this section, we study the behavior of unbounded solutions of system (2).

Theorem 14. Consider system (2). Then, for every positive solution $\left\{\left(x_{n}, y_{n}\right)\right\}$ of $(2)$ the following statements are true:

(i) let $\beta_{1}<a_{1}$ and $\beta_{2}>a_{2}+b_{2} U_{1}$; then $y_{n} \rightarrow \infty$ as $n \rightarrow \infty$;

(ii) let $\beta_{2}<a_{2}$ and $\beta_{1}>a_{1}+b_{1} U_{2}$; then $x_{n} \rightarrow \infty$ as $n \rightarrow \infty$.

Proof. (i) Suppose that $a_{1}<\beta_{1}$; then it follows from Theorem 1 that $x_{n} \leq \alpha_{1} /\left(a_{1}-\beta_{1}\right)=U_{1}, n=1,2, \ldots$. Furthermore, from system (2) it follows that

$$
\begin{aligned}
y_{n+1} & =\frac{\alpha_{2}+\beta_{2} y_{n-1}}{a_{2}+b_{2} x_{n}} \\
& \geq \frac{\alpha_{2}+\beta_{2} y_{n-1}}{a_{2}+b_{2} U_{1}} \\
& =c_{2}+d_{2} y_{n-1},
\end{aligned}
$$

where

$$
c_{2}=\frac{\alpha_{2}}{a_{2}+b_{2} U_{1}}, \quad d_{2}=\frac{\beta_{2}}{a_{2}+b_{2} U_{1}} .
$$

Consider the following second-order difference equation:

$$
w_{n+1}=c_{2}+d_{2} w_{n-1}, \quad n=0,1, \ldots
$$

The solution of (47) is given by

$$
w_{n}=\frac{c_{2}}{1-d_{2}}+r_{1} d_{2}^{n / 2}+r_{2}\left(-\sqrt{d_{2}}\right)^{n}, \quad n=1,2, \ldots,
$$

where $r_{1}, r_{2}$ depend on initial values $w_{-1}, w_{0}$. Moreover, assume that $\beta_{2}>a_{2}+b_{2} U_{1}$; that is, $d_{2}=\beta_{2} /\left(a_{2}+b_{2} U_{1}\right)>1$; then we obtain that $\left\{w_{n}\right\}$ is divergent. Hence, by comparison, we have $y_{n} \rightarrow \infty$ as $n \rightarrow \infty$.

(ii) Assume that $a_{2}<\beta_{2}$; then from Theorem 1 we obtain that $y_{n} \leq \alpha_{2} /\left(a_{2}-\beta_{2}\right)=U_{2}, n=1,2, \ldots$. Moreover, from system (2) we have

$$
\begin{aligned}
x_{n+1} & =\frac{\alpha_{1}+\beta_{1} x_{n-1}}{a_{1}+b_{1} y_{n}} \\
& \geq \frac{\alpha_{1}+\beta_{1} x_{n-1}}{a_{1}+b_{1} U_{2}} \\
& =c_{1}+d_{1} x_{n-1},
\end{aligned}
$$

where

$$
c_{1}=\frac{\alpha_{1}}{a_{1}+b_{1} U_{2}}, \quad d_{1}=\frac{\beta_{1}}{a_{1}+b_{1} U_{2}} .
$$

Next, we consider the following second-order difference equation:

$$
z_{n+1}=c_{1}+d_{1} z_{n-1}, \quad n=0,1, \ldots
$$

Then, it is easy to see that solution of (51) is given by

$$
z_{n}=\frac{c_{1}}{1-d_{1}}+r_{3} d_{1}^{n / 2}+r_{4}\left(-\sqrt{d_{1}}\right)^{n}, \quad n=1,2, \ldots,
$$

where $r_{3}, r_{4}$ depend on initial values $z_{-1}, z_{0}$. Furthermore, suppose that $\beta_{1}>a_{1}+b_{1} U_{2}$; that is, $d_{1}=\beta_{1} /\left(a_{1}+b_{1} U_{2}\right)>1$; then one has $\left\{z_{n}\right\}$ that is divergent. Hence, by comparison we have $x_{n} \rightarrow \infty$ as $n \rightarrow \infty$.

\section{Periodicity Nature of Solutions of (2)}

Theorem 15. Assume that $a_{1}>\beta_{1}$ and $a_{2}>\beta_{2}$; then system (2) has no prime period-two solutions.

Proof. On the contrary, suppose that the system (2) has a distinctive prime period-two solutions

$$
\ldots,\left(p_{1}, q_{1}\right),\left(p_{2}, q_{2}\right),\left(p_{1}, q_{1}\right), \ldots
$$

where $p_{1} \neq p_{2}, q_{1} \neq q_{2}$, and $p_{i}, q_{i}$ are positive real numbers for $i \in\{1,2\}$. Then, from system (2), one has

$$
\begin{array}{ll}
p_{1}=\frac{\alpha_{1}+\beta_{1} p_{1}}{a_{1}+b_{1} q_{2}}, & p_{2}=\frac{\alpha_{1}+\beta_{1} p_{2}}{a_{1}+b_{1} q_{1}} \\
q_{1}=\frac{\alpha_{2}+\beta_{2} q_{1}}{a_{2}+b_{2} p_{2}}, & q_{2}=\frac{\alpha_{2}+\beta_{2} q_{2}}{a_{2}+b_{2} p_{1}} .
\end{array}
$$

After some tedious calculations from (54), we obtain

$$
\begin{aligned}
p_{1}+p_{2} & =\frac{\mu+\sqrt{4 b_{2} \alpha_{1}\left(a_{1}-\beta_{1}\right)\left(a_{2}-\beta_{2}\right)+\mu^{2}}}{b_{2}\left(a_{1}-\beta_{1}\right)}, \\
p_{1} p_{2} & =\left(\frac{\mu+\sqrt{4 b_{2} \alpha_{1}\left(a_{1}-\beta_{1}\right)\left(a_{2}-\beta_{2}\right)+\mu^{2}}}{2 b_{2}\left(a_{1}-\beta_{1}\right)}\right)^{2}, \\
q_{1}+q_{2} & =\frac{\nu+\sqrt{4 b_{2} \alpha_{1}\left(a_{1}-\beta_{1}\right)\left(a_{2}-\beta_{2}\right)+v^{2}}}{b_{1}\left(a_{2}-\beta_{2}\right)}, \\
q_{1} q_{2} & =\left(\frac{\nu+\sqrt{4 b_{2} \alpha_{1}\left(a_{1}-\beta_{1}\right)\left(a_{2}-\beta_{2}\right)+v^{2}}}{2 b_{1}\left(a_{2}-\beta_{2}\right)}\right)^{2},
\end{aligned}
$$

where $\mu=\left(a_{2}-\beta_{2}\right)\left(\beta_{1}-a_{1}\right)+b_{2} \alpha_{1}-b_{1} \alpha_{2}$ and $\nu=\left(a_{2}-\right.$ $\left.\beta_{2}\right)\left(\beta_{1}-a_{1}\right)-b_{2} \alpha_{1}+b_{1} \alpha_{2}$. From (55), it follows that

$$
\left(p_{1}+p_{2}\right)^{2}-4 p_{1} p_{2}=0 .
$$

Similarly, from (56), we have

$$
\left(q_{1}+q_{2}\right)^{2}-4 q_{1} q_{2}=0 .
$$

Obviously, from (57) and (58), one has $p_{1}=p_{2}$ and $q_{1}=$ $q_{2}$, respectively, which is a contradiction. Hence, the proof is completed. 


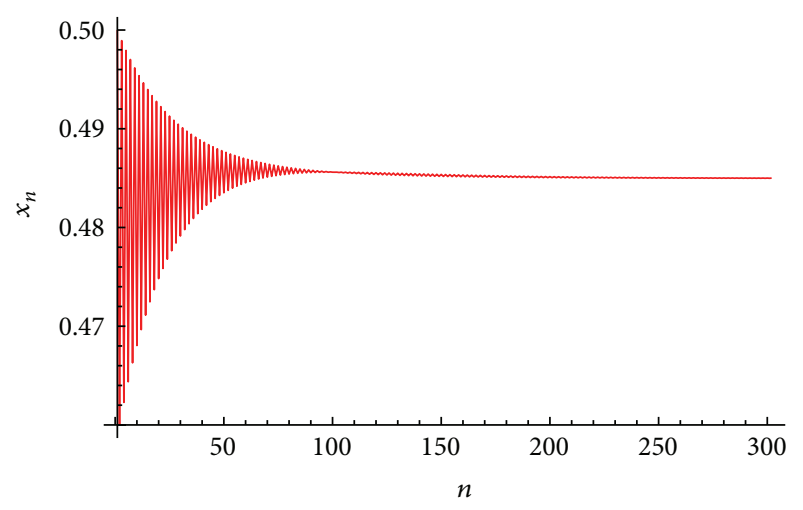

(a) Plot of $x_{n}$ for the system (59)

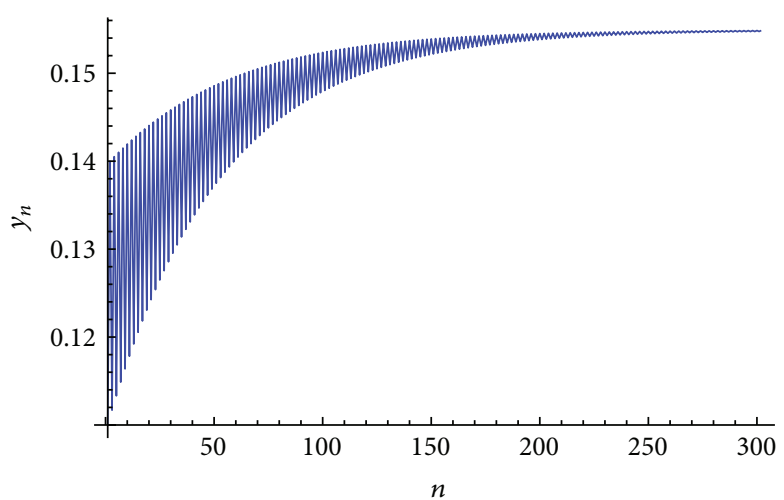

(b) Plot of $y_{n}$ for the system (59)

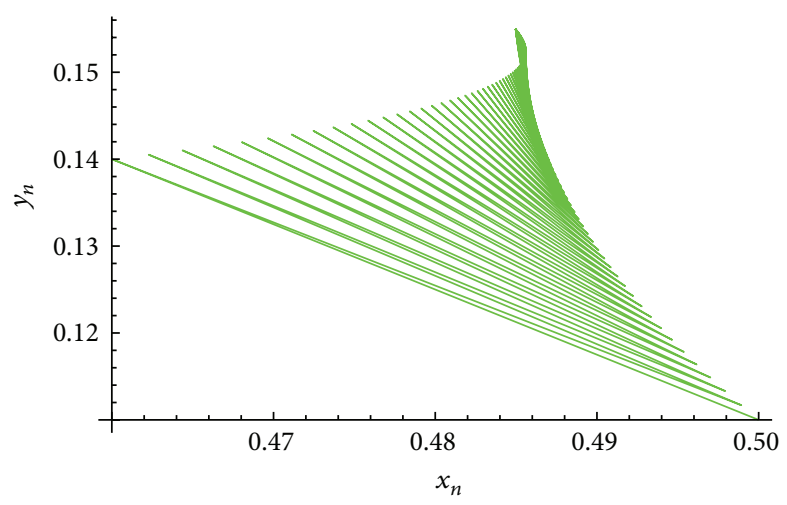

(c) An attractor of the system (59)

FIGURE 1: Plots for the system (59).

\section{Examples}

Example 1. Let $\alpha_{1}=0.5, \beta_{1}=12, a_{1}=13, b_{1}=0.2, \alpha_{2}=0.1$, $\beta_{2}=17, a_{2}=17.5$, and $b_{2}=0.3$. Then, system (2) can be written as

$$
x_{n+1}=\frac{0.5+12 x_{n-1}}{13+0.2 y_{n}}, \quad y_{n+1}=\frac{0.1+17 y_{n-1}}{17.5+0.3 x_{n}},
$$

with initial conditions $x_{0}=0.46, x_{-1}=0.5, y_{-1}=0.11$, and $y_{0}=0.14$.

In this case, the unique positive equilibrium point of the system (59) is given by $(\bar{x}, \bar{y})=(0.484974,0.154921)$. Moreover, in Figure 1, the plot of $x_{n}$ is shown in Figure 1(a), the plot of $y_{n}$ is shown in Figure 1(b), and an attractor of the system (59) is shown in Figure 1(c).

Example 2. Let $\alpha_{1}=10, \beta_{1}=1.5, a_{1}=1.6, b_{1}=0.003, \alpha_{2}=$ $12, \beta_{2}=23, a_{2}=23.1$, and $b_{2}=0.02$. Then, system (2) can be written as

$$
x_{n+1}=\frac{10+1.5 x_{n-1}}{1.6+0.003 y_{n}}, \quad y_{n+1}=\frac{12+23 y_{n-1}}{23.1+0.02 x_{n}},
$$

with initial conditions $x_{-1}=82, x_{0}=89, y_{-1}=5.9$, and $y_{0}=6$.

In this case, the unique positive equilibrium point of the system $(60)$ is given by $(\bar{x}, \bar{y})=(83.0225,6.81644)$. Moreover, in Figure 2, the plot of $x_{n}$ is shown in Figure 2(a), the plot of $y_{n}$ is shown in Figure 2(b), and an attractor of the system (60) is shown in Figure 2(c).

Example 3. Let $\alpha_{1}=3.2, \beta_{1}=8, a_{1}=8.1, b_{1}=5.5, \alpha_{2}=$ $4.2, \beta_{2}=16, a_{2}=16.1$, and $b_{2}=8.5$. Then, system ( 2 ) can be written as

$$
x_{n+1}=\frac{3.2+8 x_{n-1}}{8.1+5.5 y_{n}}, \quad y_{n+1}=\frac{4.2+16 y_{n-1}}{16.1+8.5 x_{n}},
$$

with initial conditions $x_{-1}=3.9, x_{0}=3.5, y_{-1}=0.1$, and $y_{0}=0.12$.

In this case, the unique positive equilibrium point of the system $(61)$ is given by $(\bar{x}, \bar{y})=(4.88876,0.10083)$. Moreover, in Figure 3, the plot of $x_{n}$ is shown in Figure 3(a), the plot of $y_{n}$ is shown in Figure 3(b), and an attractor of the system (61) is shown in Figure 3(c).

\section{Concluding Remarks}

In literature, several articles are related to qualitative behavior of competitive system of planar rational difference equations [20]. It is very interesting mathematical problem to study the dynamics of competitive systems in higher dimension. This work is related to qualitative behavior of competitive system of second-order rational difference equations. We have investigated the existence and uniqueness of positive steady 


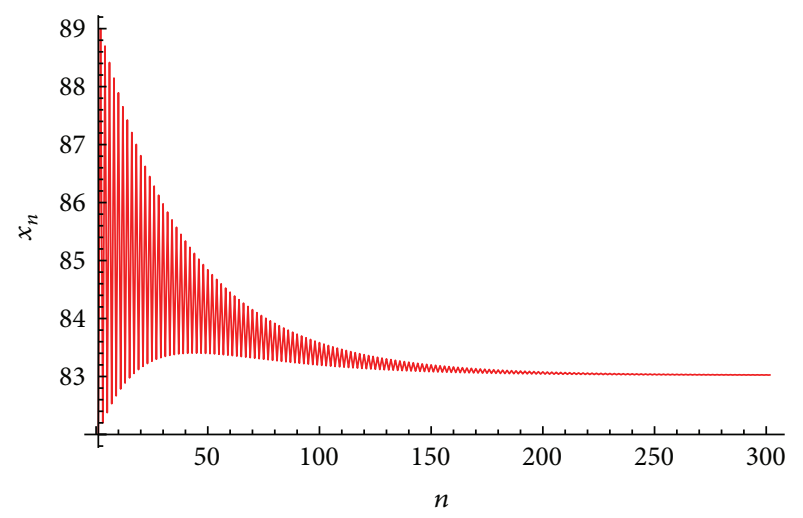

(a) Plot of $x_{n}$ for the system (60)

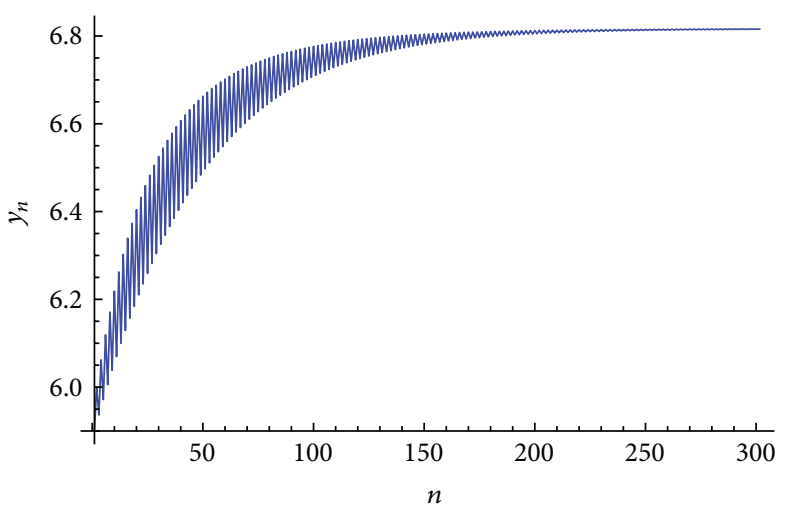

(b) Plot of $y_{n}$ for the system (60)

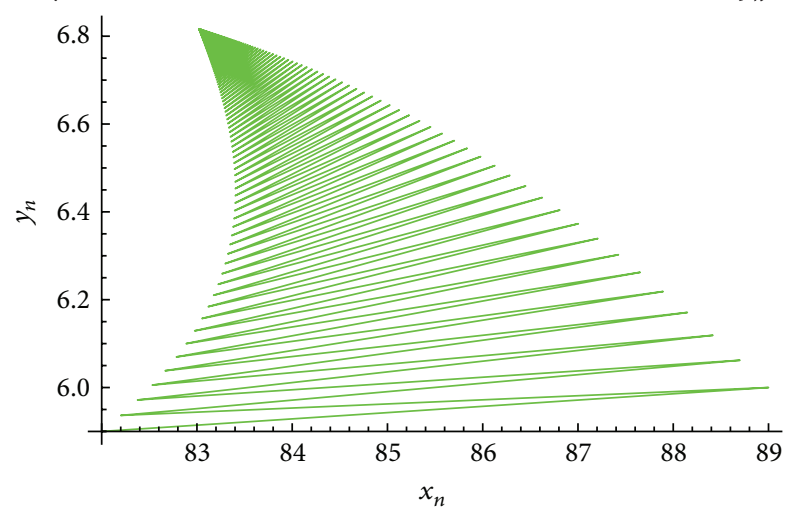

(c) An attractor of the system (60)

FIgURE 2: Plots for the system (60).

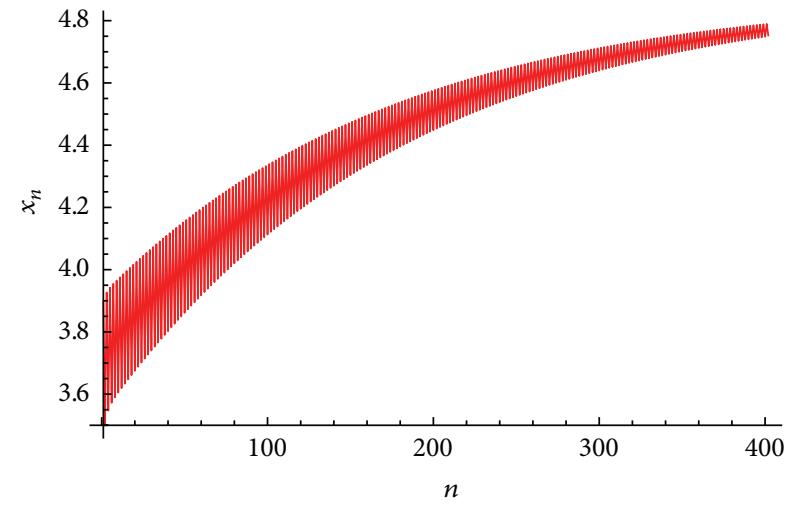

(a) Plot of $x_{n}$ for the system (61)

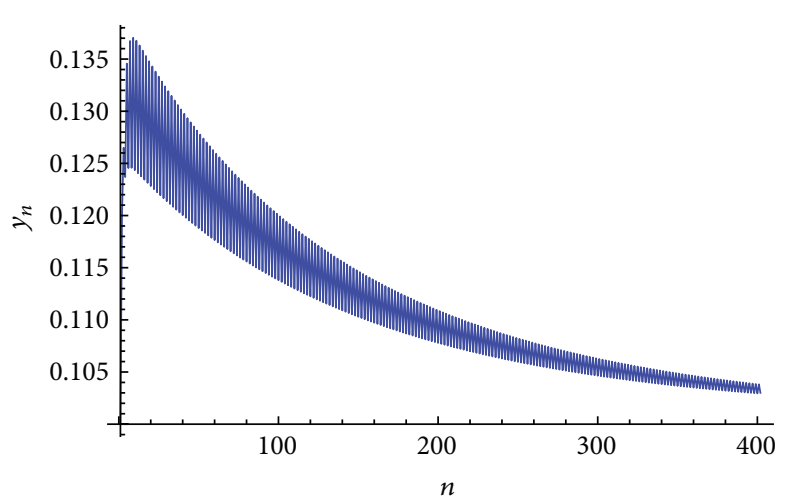

(b) Plot of $y_{n}$ for the system (61)

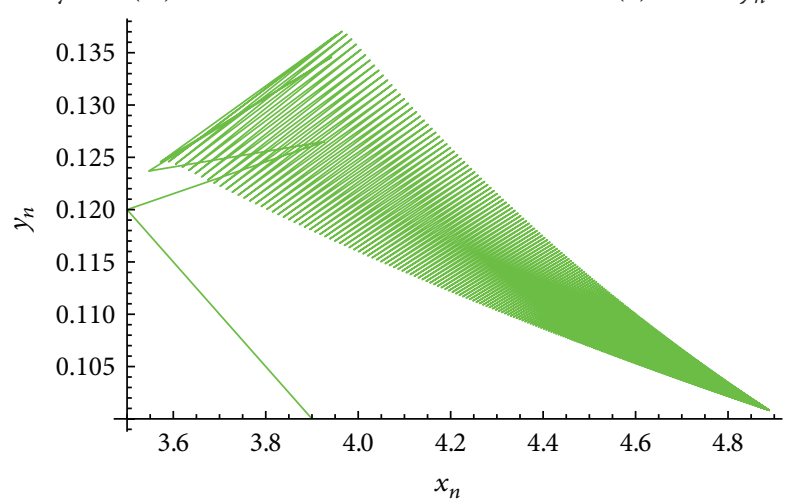

(c) An attractor of the system (61)

Figure 3: Plots for the system (61). 
state of system (2). Under certain parametric conditions the boundedness and persistence of positive solutions is proved. Moreover, we have shown that unique positive equilibrium point of system (2) is locally as well as globally asymptotically stable. Furthermore, rate of convergence of positive solutions of (2) which converge to its unique positive equilibrium point is demonstrated. Finally, existence of unbounded solutions and periodicity nature of positive solutions of this competitive system are given.

\section{Conflict of Interests}

The authors declare that they have no conflict of interests regarding the publication of this paper.

\section{Acknowledgments}

The authors thank the main editor and anonymous referees for their valuable comments and suggestions leading to improvement of this paper. For the first author, this work was partially supported by the Higher Education Commission of Pakistan.

\section{References}

[1] M. R. S. Kulenović and G. Ladas, Dynamics of Second Order Rational Difference Equations, Chapman \& Hall/CRC, Boca Raton, Fla, USA, 2002.

[2] E. A. Grove and G. Ladas, Periodicities in Nonlinear Difference Equations, vol. 4 of Advances in Discrete Mathematics and Applications, Chapman \& Hall/CRC, Boca Raton, Fla, USA, 2005.

[3] H. Sedaghat, Nonlinear Difference Equations: Theory with Applications to Social Science Models, vol. 15 of Mathematical Modelling: Theory and Applications, Kluwer Academic Publishers, Dordrecht, The Netherlands, 2003.

[4] S. Ahmad, "On the nonautonomous Volterra-Lotka competition equations," Proceedings of the American Mathematical Society, vol. 117, no. 1, pp. 199-204, 1993.

[5] X. Tang and X. Zou, "On positive periodic solutions of LotkaVolterra competition systems with deviating arguments," Proceedings of the American Mathematical Society, vol. 134, no. 10, pp. 2967-2974, 2006.

[6] Z. Zhou and X. Zou, "Stable periodic solutions in a discrete periodic logistic equation," Applied Mathematics Letters, vol. 16, no. 2, pp. 165-171, 2003.

[7] X. Liu, "A note on the existence of periodic solutions in discrete predator-prey models," Applied Mathematical Modelling, vol. 34, no. 9, pp. 2477-2483, 2010.

[8] Q. Din, "Dynamics of a discrete Lotka-Volterra model," Advances in Difference Equations, vol. 2013, article 95, pp. 1-13, 2013.

[9] Q. Din and T. Donchev, "Global character of a host-parasite model," Chaos, Solitons \& Fractals, vol. 54, pp. 1-7, 2013.

[10] Q. Din and E. M. Elsayed, "Stability analysis of a discrete ecological model," Computational Ecology and Software, vol. 4, no. 2, pp. 89-103, 2014.

[11] H. El-Metwally, E. A. Grove, G. Ladas, R. Levins, and M. Radin, "On the difference equation $x_{n+1}=\alpha+\beta x_{n-1} e^{-x_{n}}$," Nonlinear
Analysis: Theory, Methods \& Applications, vol. 47, no. 7, pp. 4623-4634.

[12] G. Papaschinopoulos, M. A. Radin, and C. J. Schinas, "On the system of two difference equations of exponential form: $x_{n+1}=a+b x_{n-1} e^{-y_{n}}, \quad y_{n+1}=c+d y_{n-1} e^{-x_{n}}$, Mathematical and Computer Modelling, vol. 54, no. 11-12, pp. 2969-2977, 2011.

[13] G. Papaschinopoulos, M. Radin, and C. J. Schinas, "Study of the asymptotic behavior of the solutions of three systems of difference equations of exponential form," Applied Mathematics and Computation, vol. 218, no. 9, pp. 5310-5318, 2012.

[14] G. Papaschinopoulos and C. J. Schinas, "On the dynamics of two exponential type systems of difference equations," Computers \& Mathematics with Applications, vol. 64, no. 7, pp. 2326-2334, 2012.

[15] Q. Din, "Global stability of a population model," Chaos, Solitons \& Fractals, vol. 59, pp. 119-128, 2014.

[16] A. Q. Khan, Q. Din, M. N. Qureshi, and T. F. Ibrahim, "Global behavior of an anti-competitive system of fourth-order rational difference equations," Computational Ecology and Software, vol. 4, no. 1, pp. 35-46, 2014.

[17] T. F. Ibrahim and Q. Zhang, "Stability of an anti-competitive system of rational difference equations," Archives des Sciences, vol. 66, no. 5, pp. 44-58, 2013.

[18] C. H. Gibbons, M. R. S. Kulenović, and G. Ladas, "On the recursive sequence $x_{n+1}=\left(\alpha+\beta x_{n-1}\right) /\left(\gamma+x_{n}\right)$," Mathematical Sciences Research Hot-Line, vol. 4, no. 2, pp. 1-11, 2000.

[19] M. Pituk, "More on Poincaré's and Perron's theorems for difference equations," Journal of Difference Equations and Applications, vol. 8, no. 3, pp. 201-216, 2002.

[20] M. Garić-Demirović, M. R. S. Kulenović, and M. Nurkanović, "Global behavior of four competitive rational systems of difference equations in the plane," Discrete Dynamics in Nature and Society, vol. 2009, Article ID 153058, 34 pages, 2009. 


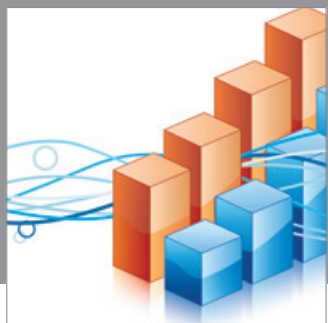

Advances in

Operations Research

mansans

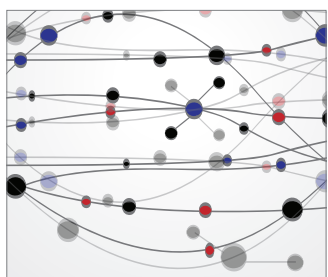

The Scientific World Journal
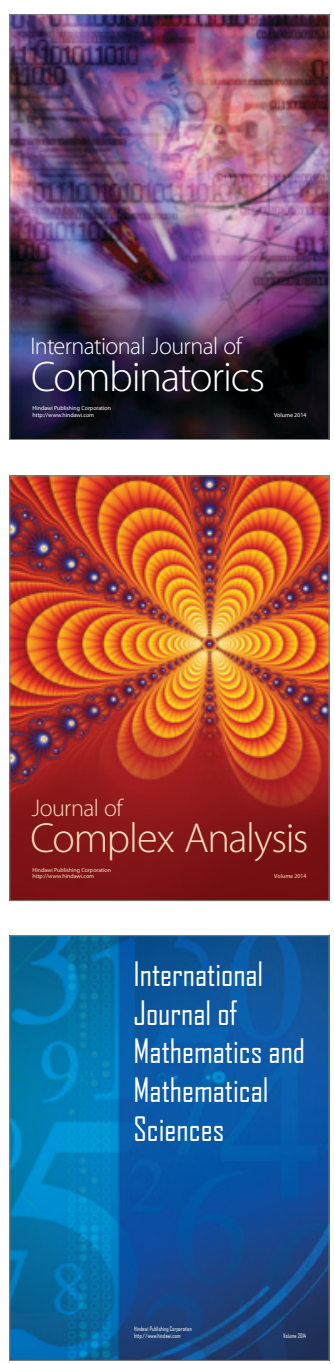
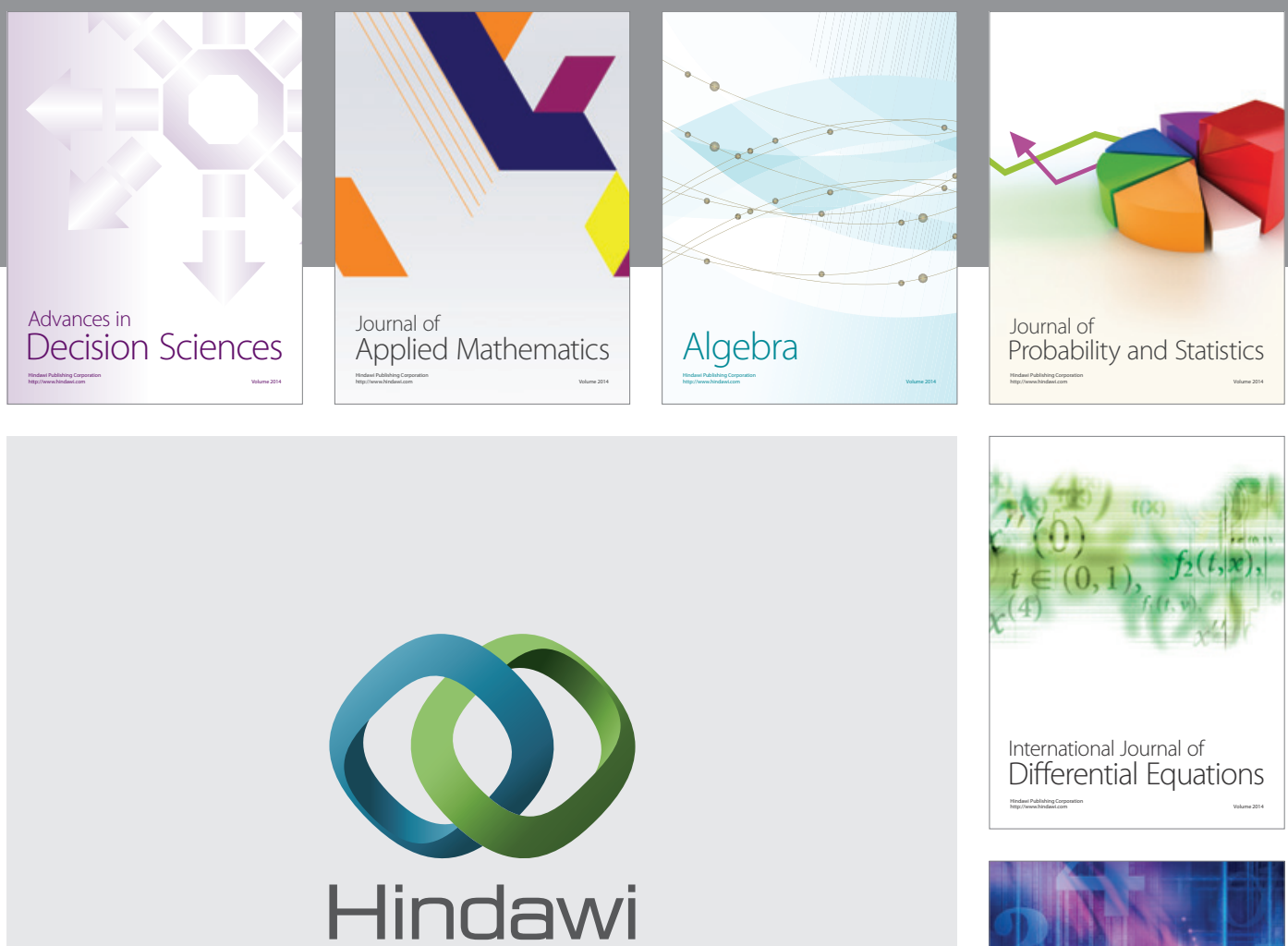

Submit your manuscripts at http://www.hindawi.com
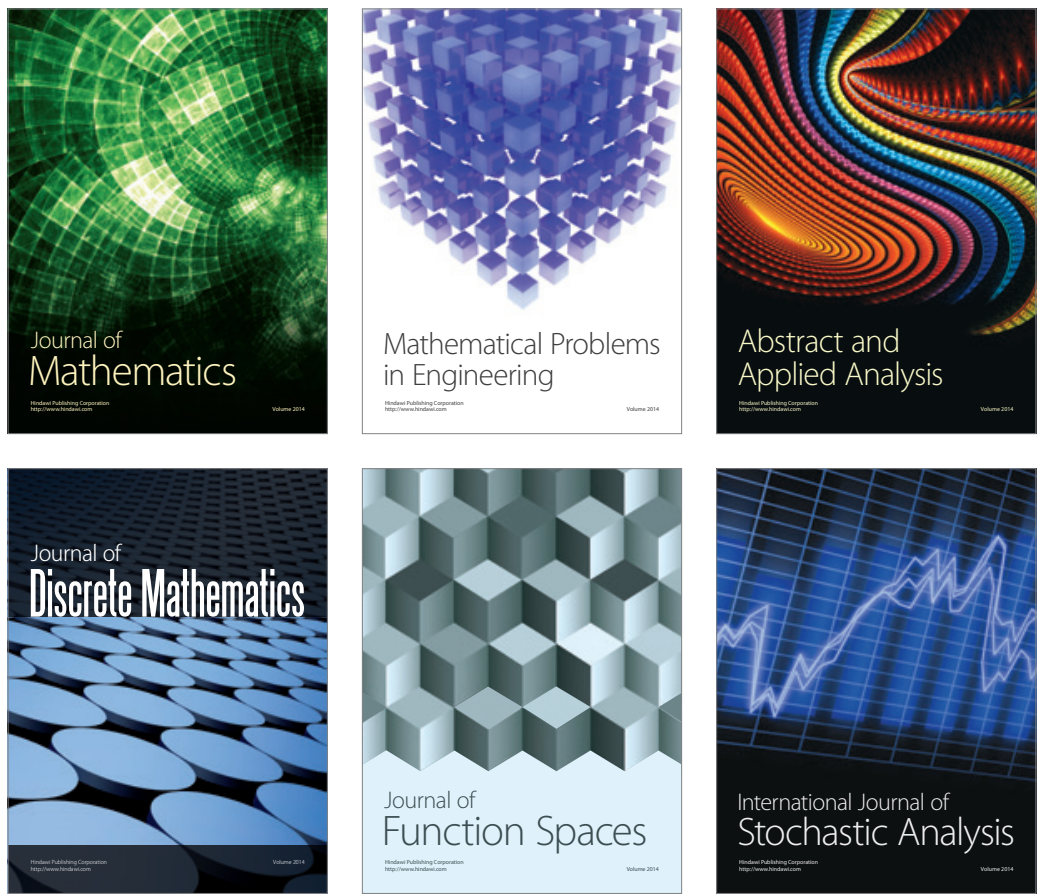

Journal of

Function Spaces

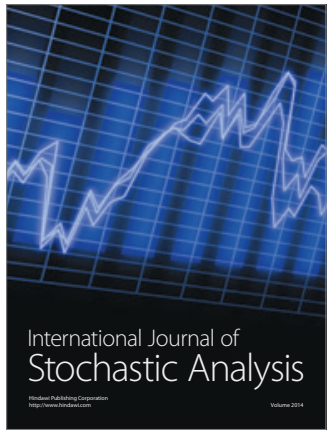

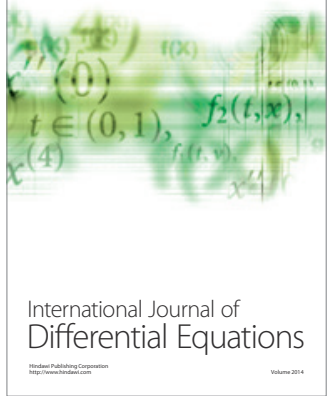
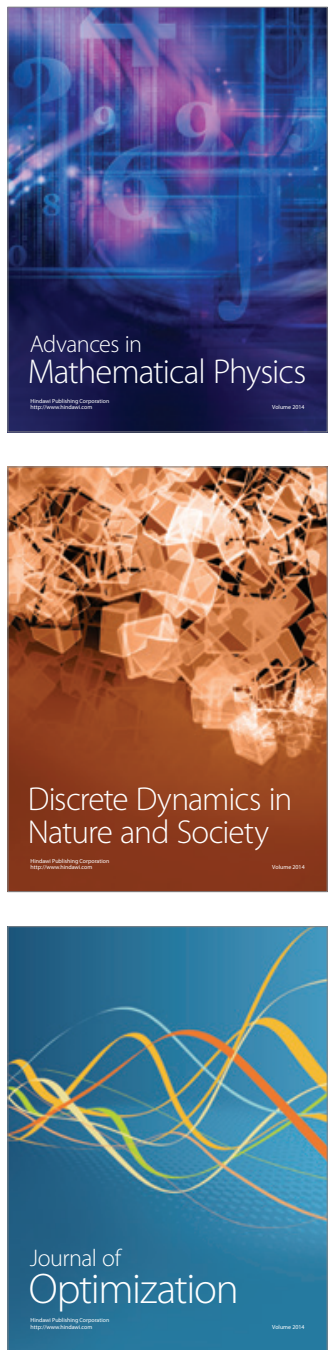